\title{
Selection, Identification, and Application of Aptamers against Agaricus bisporus Lectin to Establish an Aptamer-AuNPs Colorimetric Method for Detection of ABL
}

\author{
Hao Gao $\mathbb{D},{ }^{1}$ Yiling Tian, ${ }^{1}$ Min Zhang, ${ }^{2}$ Jianhui Liu $\mathbb{D},{ }^{1}$ Yaowu Yuan $\mathbb{D},{ }^{1}$ Jianxin Tan $\mathbb{D}^{1}$ \\ and Aijin $\mathrm{Ma} \mathbb{1}^{3}$ \\ ${ }^{1}$ College of Food Science and Technology, Hebei Agricultural University, No. 289 Lingyusi Street, Baoding 071001, Hebei, China \\ ${ }^{2}$ Shijiazhuang Junlebao Dairy Co., Ltd., No. 36 Shitong Road, Shijiazhuang 050221, Hebei, China \\ ${ }^{3}$ Beijing Technology and Business University, Beijing, No. 11 Fucheng Road, Beijing 100048, China
}

Correspondence should be addressed to Jianxin Tan; jianxintan@sina.com and Aijin Ma; maaj@btbu.edu.cn

Received 8 August 2020; Revised 29 August 2020; Accepted 4 September 2020; Published 22 October 2020

Academic Editor: Wen yi Kang

Copyright (c) 2020 Hao Gao et al. This is an open access article distributed under the Creative Commons Attribution License, which permits unrestricted use, distribution, and reproduction in any medium, provided the original work is properly cited.

\begin{abstract}
Agaricus bisporus lectin (ABL), which is one of the antinutritional factors in A. bisporus, is an important allergen and harmful to human health. Due to the shortcomings of the current detection methods, it is extremely urgent to establish a rapid and sensitive detection method for ABL in foods. To isolate the ssDNA aptamer of ABL, 13 rounds of subtractive systematic evolution of ligands by exponential enrichment (SELEX) selection were carried out. As a result, six candidate aptamers were selected and further examined for their binding affinity and specificity by enzyme-linked aptamer method. One aptamer (seq-41) against ABL with a high affinity and specificity was isolated and demonstrated to be the optimal aptamer whose dissociation constant reaches the nanomolar level, $K d=31.17 \pm 0.1070 \mathrm{nM}$. Based on seq-41, an aptamer-AuNPs colorimetric method was established to detect ABL with a linear range of $0.08 \sim 1.70 \mu \mathrm{g} / \mathrm{mL}$ and the detection limit is $0.062 \mu \mathrm{g} / \mathrm{mL}$. This study provides a novel aptamer-AuNPs colorimetric method with high sensitivity and specificity for detection of ABL and a novel strategy for development of detection method of fungal or plant allergens.
\end{abstract}

\section{Introduction}

Agaricus bisporus, also known as button mushroom, white mushroom, or champignon, is one of the most widely cultivated and consumed edible mushrooms in the world [1]. As one of the most studied bioactive proteins [2-4], A. bisporus lectin (abbreviated $\mathrm{ABL}$ ) is a $64 \mathrm{KDa}$ glycoprotein composed of four subunits [5] and has binding specificity to Gal $(\beta-1,3)$ GalNAc [6]. On the one hand, lectins display antifungal and immunomodulatory activities [7]. On the other hand, lectins have blood coagulation activity and the antinutritional effects on animals [8] because they are ones of the components formed in the long-term evolution of plants to resist diseases, insect pests and animal digestion. These lectins may contribute to the development of multiple food sensitivities, food allergies, and autoimmune conditions [9]. Therefore, the establishment and development of methods for rapid detection and identification of allergens in food (e.g., lectins) can effectively prevent people from allergies, which is very important for health.

At present, the common qualitative and quantitative assays of the lectin from plant and fungi are generally based on the agglutination assay and immunoassay. For example, the hemagglutination activity test can be served to monitor and quantify lectin activity at each step during purification [10]. In addition, based on the protein properties of lectins and immunological techniques, a variety of assay methods for quantifying lectin activity have been developed, such as electrochemical immunoassay (ECIA) [11] and enzymelinked immunosorbent assay (ELISA) [12, 13]. Comparatively, the procedure of hemagglutination test is relatively simple and easy to be operated. However, the same lectin 
may show different agglutination activity to red blood cells from the different kinds of animals, and its accuracy and sensitivity are relatively low. Accordingly, this method may be more suitable for semiquantitative or qualitative analysis of lectin content. Except for the hemagglutination test, other detection methods have the disadvantages of cumbersome steps and complex operation. Hence, it is particularly important to develop a new convenient and sensitive method for the detection of lectin in order to provide technical support for ensuring food safety and human health.

Aptamers are short single-stranded DNA or RNA with high affinity and specificity to the target molecule [14] and are screened by systematic evolution of ligands by exponential enrichment (SELEX) technology $[14,15]$. The concept of aptamer was first proposed by Ellington [16] and Tuerk [17]. The SELEX technology has been widely explored and used in many fields during the past 30 years. Aptamers are also called "chemical antibodies" because their characters and applications are similar to antibody [18]. However, compared with antibodies, aptamers have many advantages, such as a wide range of target molecules, short preparation cycle, strong specificity, high affinity, lower immunogenicity, and lower cost of synthesis [19]. As a new type of specific recognition probe, aptamer provides a sensitive and specific optional mode for the development of the new detection methods in the field of food safety, for example, detection of biological toxins [20, 21], pathogenic bacteria [20], antibiotics [22], and heavy metals [23]. Colloidal gold, as a new type of nanomaterials, is popularly used as biological and chemical sensors owing to its merits of easy labeling, surface plasma resonance effect, and other chemical and optical properties [24]. Particularly, the gold nanoparticle-based colorimetric assay is widely used in environmental monitoring, public health, and disease diagnosis because of their simple, cost-effective fabrication and ease of use [24]. Combined usage of aptamer and gold nanoparticle-based colorimetric assay displays both advantages and provides a much more powerful and useful strategy for developing usable methods and their applications [25].

The study aims to explore a simple, easy, and sensitive method for the determination of Agaricus bisporus lectin. We use subtractive-SELEX technology to obtain high-affinity aptamers after 13 selection rounds. Using the selected aptamer as a specific recognition probe and AuNPs as an indicator, a visual detection method of ABL was established to effectively monitor the lectin content of A. bisporus products. The results of this study will provide a new reference for developing detection methods of other allergens from fungi or plants.

\section{Materials and Methods}

2.1. Materials. A single-strand DNA (ssDNA) library (5'ATAGGAGTCACGACGACCAG-N40-TATGTGCGTCTACCTCTTGA- $3^{\prime}$ ) and primers (forward primer1/FP1: $5^{\prime}$ ATAGGAGTCACGACGACCAG-3', reverse primer1/RP1: $5^{\prime}$-TCAAGAGGTAGACGCACATA- $3^{\prime}$, and reverse primer2/RP2: $\quad 5^{\prime}$-biotin-TCAAGAGGTAGACGCACATA$\left.3^{\prime}\right)$ for the amplifying and screening of ABL aptamer were designed and synthesized by Sangon Bioengineering (Shanghai) Co. Ltd. China. $2 \times$ Es Taq Master Mix (Dye) was purchased from Beijing ComWin Biotech Co., Ltd, China. Agaricus bisporus lectin FL-1420 was purchased from Vector Laboratories Inc. Bovine serum albumin, acrylamide, TEMED, streptavidin-horseradish peroxidase (HRP-SA), and $3,3^{\prime}, 5,5^{\prime}$-tetramethylbenzidine (TMB) were purchased from Beijing Solarbio Science \& Technology Co., Ltd. China. Streptavidin magnetic beads were ordered from Suzhou Beaver Biomedical Engineering Co., Ltd. China. Nucleic acid coprecipitation reagent kits were bought from Takara Biomedical Technology Co., Ltd. All other reagents were from Sinopharm Chemical Reagent Company, China.

2.2. Aptamer Selection. The aptamer selection procedures followed the protocol of Du et al. [26] with some minor changes. First, ABL or BSA was adsorbed onto the wells of 96-well plates. In detail, at the first round, the plates were coated with $200 \mu \mathrm{L} /$ well of $50 \mu \mathrm{g} / \mathrm{mL}$ of ABL or BSA dissolved in binding buffer (20 mM HEPES, $120 \mathrm{mM} \mathrm{NaCl}$, $5 \mathrm{mM} \mathrm{KCl}, 1 \mathrm{mM} \mathrm{CaCl}$, and $1 \mathrm{mM} \mathrm{MgCl}_{2}, \mathrm{pH} 7.4$ ) and incubated overnight at $4^{\circ} \mathrm{C}$. After washing three times with the binding buffer, the plates were blocked with $3 \%(\mathrm{w} / \mathrm{v})$ BSA solution for $2 \mathrm{~h}$ at $37^{\circ} \mathrm{C}$ and then the unbound BSA was removed. Next, $2 \mathrm{nM}$ of the random ssDNA pool was prepared in $100 \mu \mathrm{L}$ of binding buffer and denatured for $5 \mathrm{~min}$ at $95^{\circ} \mathrm{C}$, cooled for $5 \mathrm{~min}$ at $4^{\circ} \mathrm{C}$, and then incubated for $10 \mathrm{~min}$ at room temperature. The denatured ssDNA pool was added into the BSA coated wells and incubated for $40 \mathrm{~min}$ at $37^{\circ} \mathrm{C}$ to eliminate the ssDNAs that were able to bind BSA. The unbound ssDNAs were collected, added into the ABL-coated wells, and incubated for $60 \mathrm{~min}$ at $37^{\circ} \mathrm{C}$. After incubation, the wells were washed three times with binding buffer containing $0.05 \%$ Tween-20 to remove unbound ssDNAs. The remaining ssDNAs that bound to ABL were incubated in elution buffer $(20 \mathrm{mM}$ Tris- $\mathrm{HCl}, 4 \mathrm{mM}$ guanidine isothiocyanate, and $1 \mathrm{mM}$ DTT, $\mathrm{pH}$ 8.3) for $10 \mathrm{~min}$ at $90^{\circ} \mathrm{C}$ and then collected and recovered by alcohol precipitation. The enrichment of ABL binding ssDNAs was monitored by the recovery of ssDNA. The purified ssDNA were amplified by PCR $(2 \times$ Es Taq Master Mix (Dye) $12.5 \mu \mathrm{L}$, FP1 $(20 \mu \mathrm{M}) 0.5 \mu \mathrm{L}, \mathrm{RP} 2(20 \mu \mathrm{M}) 0.5 \mu \mathrm{L}$, ssDNA Template $2.0 \mu \mathrm{L}, \mathrm{ddH}_{2} \mathrm{O} 9.5 \mu \mathrm{L}$ in a total volume of $25 \mu \mathrm{L}$ ). PCR was performed following the program: predenaturtion at $95^{\circ} \mathrm{C}$ for $5 \mathrm{~min}$, followed by 20 cycles of $95^{\circ} \mathrm{C}$ for $30 \mathrm{~s}, 58^{\circ} \mathrm{C}$ for $30 \mathrm{~s}$, and $72^{\circ} \mathrm{C}$ for $30 \mathrm{~s}$ with a final extension at $72^{\circ} \mathrm{C}$ for $5 \mathrm{~min}$. After amplification, PCR products were denatured using $150 \mathrm{mM} \mathrm{NaOH}$ solution, and the unlabeled ssDNA strands were separated using streptavidin-coated magnetic beads and concentrated by ethanol precipitation. The isolated ssDNA strands were subjected to the next round of SELEX until the thirteenth round of selections were completed.

2.3. Aptamer Sequencing and Analysis. The entire enriched ssDNA pool of the last SELEX round was amplified by PCR and ligated into pMD18-T vector. The resultant ligation products were transformed into $E$. coli $\mathrm{DH} 5 \alpha$ competent 
cells. The plasmids containing the selected candidate aptamers harbored in the positive colonies were extracted and sequenced by Bejing Genomics Institute (BGI), China. Multisequence alignment and phylogenetic tree of the enriched sequences were performed with Clustal X 2.1 and MEGAX 10.0.5 software. Secondary structure and the free energy prediction of the enriched sequences were conducted by using RNA Structure Network Service (http://rna.urmc. rochester.edu/RNAstructureWeb/). Based on the primary sequences, secondary structure of the candidate aptamers, and the phylogenetic tree, six representative candidate aptamers were selected for subsequent affinity and specificity analysis.

2.4. Evaluation of the Aptamer Binding Affinity and Specificity. For determination of affinity, firstly, $100 \mu \mathrm{L}$ of the biotinlabeled candidate aptamers in different concentrations (10, $50,100,200,300$, and $400 \mathrm{nM}$ ) were denatured at $95^{\circ} \mathrm{C}$ for $5 \mathrm{~min}$ and then immediately cooled at $4^{\circ} \mathrm{C}$ for $5 \mathrm{~min}$, followed by incubation for $10 \mathrm{~min}$ at room temperature. Next, the solution was transferred into the ABL precoated wells and incubated at $37^{\circ} \mathrm{C}$ for $1 \mathrm{~h}$. Each well was washed three times with $200 \mu \mathrm{L}$ washing buffer (binging buffer plus $0.05 \%$ Tween-20) and then incubated with $100 \mu \mathrm{L}$ streptavidin-horseradish peroxidase (dilution $1: 5000$ ) at $37^{\circ} \mathrm{C}$ for $30 \mathrm{~min}$. After washing three times with washing buffer, $100 \mu \mathrm{L}$ substrate (TMB) was added into each well and incubated for $10 \mathrm{~min}$ at room temperature, and then, $50 \mu \mathrm{L}$ of $2 \mathrm{M} \mathrm{H}_{2} \mathrm{SO}_{4}$ was added to stop the reaction. The optical density (OD) at $450 \mathrm{~nm}$ was measured using BSA precoated well as blank. The dissociation constant ( $K_{d}$ value) of aptamer, which reflects the aptamer affinity, was analyzed with GraphPad Prism software (version 6.01, Windows) and calculated according to the equation $Y=B_{\max }^{*} \mathrm{X} /$ $\left(K_{d}+X\right)$, where $B_{\max }$ is the number of maximum binding sites, $X$ is the aptamer concentration, and $Y$ is the absorbance value.

To evaluate the specificity of the selected aptamer candidates, $1 \mu \mathrm{g} \mathrm{ABL}$, lectin extract from fresh A. bisporus fruit, and BSA were precoated in a 96-well microplate and incubated with $200 \mathrm{nM}$ biotinylated aptamer for binding with ABL. The following steps were the same as that of affinity test. Finally, the amount of the binding aptamers is determined by absorbance value at $450 \mathrm{~nm}$.

\subsection{Establishment of an Aptamer-AuNPs Colorimetric Method for Detection of $A B L$}

2.5.1. Preparation of AuNPs. AuNPs were prepared by sodium citrate reduction method created by Frens [27].

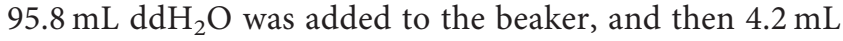
$1 \%$ chloroauric acid solution was added and heated to boiling, $10 \mathrm{~mL} 1 \%$ sodium citrate solution was quickly added and heated to boiling for $20 \mathrm{~min}$, and then the solution was continually stirred. The AuNPs solution was finally obtained after cooling to room temperature and the solution color turned to wine-red.
2.5.2. Optimization of $\mathrm{NaCl}$ Concentration. $20 \mu \mathrm{L}$ of $\mathrm{NaCl}$ solution in different concentrations $(0,50,100,150,200,250$, 300,350 , and $400 \mathrm{mM}$ ) was mixed with $100 \mu \mathrm{L}$ of colloidal gold solution, respectively, and incubated at $37^{\circ} \mathrm{C}$ for $10 \mathrm{~min}$. The color change was observed and measured over the wavelength ranging from $400 \mathrm{~nm}$ to $700 \mathrm{~nm}$.

2.5.3. Optimization of Aptamer Concentration. $100 \mu \mathrm{L}$ of different concentrations of the aptamer solution $(0,0.5,1.0$, $2.0,4.0,6.0,8.0$, and $10.0 \mu \mathrm{M})$ was added to $100 \mu \mathrm{L}$ of colloidal gold solution, mixed, and incubated at $37^{\circ} \mathrm{C}$ for $3 \mathrm{~h}$, and finally $20 \mu \mathrm{L} 250 \mathrm{mM} \mathrm{NaCl}$ was added. The color change was observed and the absorbance value was measured at $520 \mathrm{~nm}$.

2.5.4. Quantitative Detection of ABL and the Establishment of the Standard Curve. $100 \mu \mathrm{L}$ of $2 \mu \mathrm{M}$ aptamer solution was added to $100 \mu \mathrm{L}$ colloidal gold solution and incubated at $37^{\circ} \mathrm{C}$ for $3 \mathrm{~h}$ in a shaker. Then, different concentrations of ABL standard samples $(0,0.043,0.087,0.17,0.30,0.35,0.39$, $0.87,1.28,1.7$, and $2.0 \mu \mathrm{g} / \mathrm{mL}$ ) were added and incubated at $37^{\circ} \mathrm{C}$ for $1 \mathrm{~h}$. Finally, after adding $20 \mu \mathrm{L}$ of $250 \mathrm{mM} \mathrm{NaCl}$, the color change was observed and the ratio of the absorbance value at $620 \mathrm{~nm}$ to $520 \mathrm{~nm}$ was measured. A standard curve was generated using GraphPad Prism 6.01 software.

2.5.5. Specificity Tests. To evaluate the specificity of the aptamer, the same procedures were performed as mentioned in Section 2.5.4 with the samples of standard ABL, Agaricus bisporus extract, BSA, Flammulina velutipes extract, and Lentinula edodes extract in the reaction mix, and the specificity was determined by the ratio of the absorbance value at $620 \mathrm{~nm}$ to $520 \mathrm{~nm}$. Fresh lectin extracts were prepared as follows: fresh fruiting bodies of A. bisporus, $F$. velutipes, and $L$. edodes were mixed with $0.05 \mathrm{M}$ PBS $(\mathrm{pH}$ 7.2), ground in a blender, and soaked overnight at $4^{\circ} \mathrm{C}$. The homogenate was filtered through cheesecloth, and the filtrate was centrifuged at $14000 \times \mathrm{g}$ for $15 \mathrm{~min}$ at $4^{\circ} \mathrm{C}$. The supernatants were collected as fresh A. bisporus, F. velutipes, and $L$. edodes extract samples.

\section{Results and Discussion}

3.1. Selection of the Specific Aptamers against $A B L$. Proteins can be physically adsorbed by the interaction between their hydrophobic groups and the hydrophobic groups on the surface of polystyrene microplate. As glycoprotein, ABL can fix itself on the microplate by hydrophobic interaction. Taking advantage of this feature, we can separate and isolate the ssDNAs which stably bind to the $\mathrm{ABL}$ from the ssDNAs that do not bind by repeatedly washing plates. The application of subtractive-SELEX allows removing nonspecific binding ssDNAs before each binding to the target [28]. This negative screening step greatly improves screening efficiency [29]. By using these two strategies, the selection of ABL aptamer was performed. 
To select the specific aptamers against $\mathrm{ABL}$, we first designed a ssDNA library containing a central randomized sequence of 40 nucleotides flanked by defined primerbinding sites of 20 nucleotides for PCR amplification. In the first round of SELEX selection, $2 \mathrm{nmol} \mathrm{ssDNAs} \mathrm{were} \mathrm{fixed}$ in the wells of the microplate to ensure that there was the sufficient diversity of oligonucleotide sequences in the initial selection system. In subsequent steps, gradually reducing the amount of ssDNA pool and ABL is conducive to improve the screening efficiency of $\mathrm{ABL}$ aptamers with high affinity. Table 1 listed the amount of ssDNA and ABL input during each round of aptamer-selection procedures.

As shown in Figure 1(a), at the first seven rounds of selection, the ssDNA recovery rate of each round obviously increased. After that, the recovery rates showed gently increase trend and reached up to the highest one at the end round. These results indicated that the specific ssDNAs binding to $A B L$ were effectively enriched and the selection strategy was practicable. The bands around $80 \mathrm{bp}$ (Figure 1(b)), which were recovered from each selection round, indicated that the products of each selection round were correct and sufficient.

3.2. Sequence Analysis and Characterization of the Selected Aptamers against $A B L$. The products of the final SELEX round were cloned and sequenced. A total of 43 sequences were obtained. Among them, seq-1 appeared the highest frequency with nine repeats. For seq- 2 to seq- 6 , each of them had two repeats. The rest only appeared once. The existence of high-frequency sequences provided evidence that the selection method served in this study was able to enrich aptamers of ABL with high efficiency. The phylogenetic tree was constructed according to the sequence homology comparison (Figure 2). As shown in Figure 2, the seq-2, seq26 , seq-32, seq-36, seq-38, and seq-39 were in a group and formed a large branch since they have relatively higher polymorphism. The rest 37 sequences clustered into another large group with much more homology and conserved sequences of the primary sequence motifs.

According to the comparison of the secondary structure and the minimum free energy, the isolated sequences were classed into six categories. A representative sequence from each group was picked out to generate diagram of their secondary structure (Figure 3). All the secondary structures contain closed or open loop, hairpin, and cruciform structure, which may form the complex three-dimensional structures and bind to the target via noncovalent bonds interaction with the target. The different secondary structure and the conformation changes affected by different environments may influence the affinity of aptamer to the target due to the variations of hydrophobic interactions, hydrogen bonding, and salt linkages formed between them [30]. The sequences of the representative candidate aptamers, their copy number out of the 43 cloned sequences, and the free energy were listed in Table 2.

3.3. Determination of the Affinity and Specificity of the $A B L$ Candidate Aptamers against $A B L$. The enzyme-linked
TABLE 1: The amount of ABL and ssDNA pool input in each round of SELEX selections.

\begin{tabular}{lcc}
\hline SELEX rounds & ABL $(\mu \mathrm{g})$ & Aptamer $(\mathrm{pmol})$ \\
\hline 1 & 10 & 2000 \\
2 & 5 & 500 \\
3 & 5 & 300 \\
4 & 5 & 200 \\
5 & 4 & 150 \\
6 & 4 & 150 \\
7 & 4 & 150 \\
8 & 2 & 100 \\
9 & 2 & 100 \\
10 & 2 & 100 \\
11 & 1 & 80 \\
12 & 1 & 80 \\
13 & 1 & 65 \\
\hline
\end{tabular}

aptamer method was used to determine the affinity of the six candidate aptamers picked out from the 43 sequences. The dissociation constant $(\mathrm{Kd})$ of the candidate aptamers was calculated by nonlinear regression analysis (Table 3 ). The binding affinity of aptamer has a high correlation with $K_{d}$ value, which means $K_{d}$ value reflects the aptamer affinity. The $K_{d}$ values of the six aptamers were between 31 and $135 \mathrm{nM}$, indicating that all the selected candidate aptamers exhibit a high affinity towards ABL although their $K_{d}$ values are more than four times different.

The nonlinear saturation curves of the six candidate aptamers were fit and generated by Graphpad Prism 6.01 software (Figure 4). As shown in Figure 4, when the aptamer concentration increased to $200 \mathrm{nM}$, the absorbance value tended to be stable, implying that the binding reaction reaches saturation. Among the six candidates, since the $K_{d}$ values of seq- 1 and seq- 41 were relatively smaller, these two candidate aptamers were selected to further test the specificity of them.

Figure 5 showed the specificity of seq- 1 and seq- 41 to the standard ABL, the Agaricus bisporus extract, and BSA (negative control). The results revealed that the absorbance values of seq- 1 and seq- 41 were significantly higher than that of BSA, which demonstrated that both candidate aptamers had high specificity to both standard ABL and Agaricus bisporus extract. Comparatively, seq- 41 was the best one of $A B L$ aptamer due to the $A b_{450 \mathrm{~nm}}$ value of seq- 41 was higher than that of seq-1. Thus, considering the affinity and specificity results, seq-41 was verified to be the best aptamer against ABL.

\subsection{Establishment of an Aptamer-AuNPs Colorimetric Method for Detection of $A B L$}

3.4.1. Optimization of $\mathrm{NaCl}$ Concentration. Negatively charged AuNPs usually aggregates under the action of electrostatic force when the salt solution, for example, $\mathrm{NaCl}$, is added [31]. The concentration of sodium chloride added directly affects the degree of AuNPs aggregation and will further interfere with the interaction between aptamer and colloidal gold. Therefore, in order to obtain the detection 


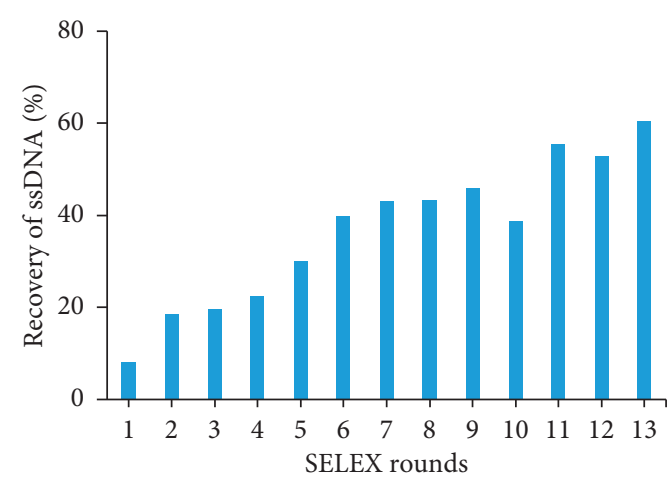

(a)

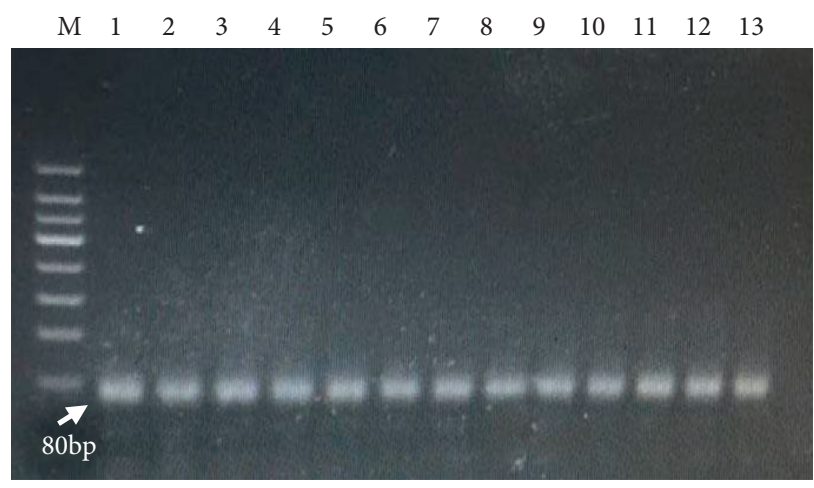

(b)

FIGURE 1: The recovery rate and the products of each SELEX selection round for ABL aptamer. (a) The recovery rate of each SELEX round. After 13 rounds of screening, with the increase of the number of screening rounds, the recovery rate was increasing overall and tended to be stable. (b) Verification of the products of each SELEX round by PCR, M 50 bp DNA ladder; 1-13: SELEX round numbers.

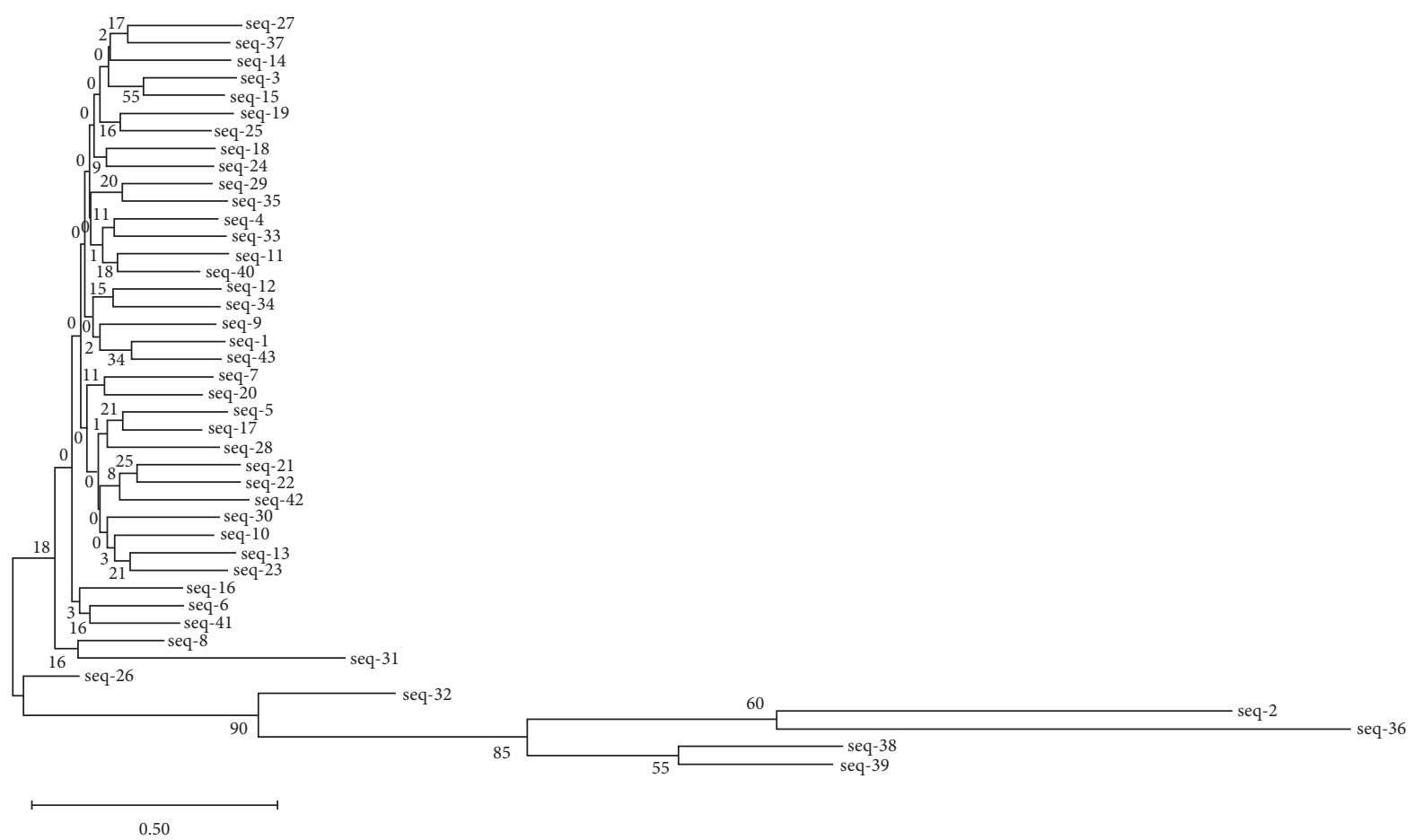

FIGURE 2: The phylogenetic tree of the cloned 43 aptamers. The phylogenetic tree was constructed by Clustal X 2.1 and MEGAX 10.0.5.

results with high sensitivity and wide detection range, the optimization of $\mathrm{NaCl}$ concentration is particularly important. As shown in Figure 6, along with the salt concentration increasing in the range from 0 to $400 \mathrm{mM}$, the color of colloidal gold solution changed from red to purple and finally to gray. Meanwhile, the $A b_{520}$ value continued to decline and finally stabilized, indicating that the colloidal gold gradually accumulated under the action of salt. When the salt concentration is between 50 and $100 \mathrm{mM}$, the red color was gradually getting lighter and the precipitation was not observed. If the salt concentration increased from $150 \mathrm{mM}$ to $200 \mathrm{mM}$, the colloidal gold solution turned purple and most of the AuNPs deposited. When the salt concentrations were in the range of $250-400 \mathrm{mM}$, the color of the colloidal gold solution became gray and the AuNPs were completely precipitated. Combined with the above observed results, the salt concentration at $250 \mathrm{mM}$ was selected and served for subsequent test.

\subsubsection{Optimization of Aptamer Concentration.} Oligonucleotides, like aptamer, can bind to colloidal gold [32], which can protect gold nanoparticles from aggregation under high salt conditions. However, when the amount of aptamers is too high, the gold nanoparticles will not agglomerate in high concentration salt solution and the color will not change obviously, which will eventually lead to elevating the detection limit. As shown 

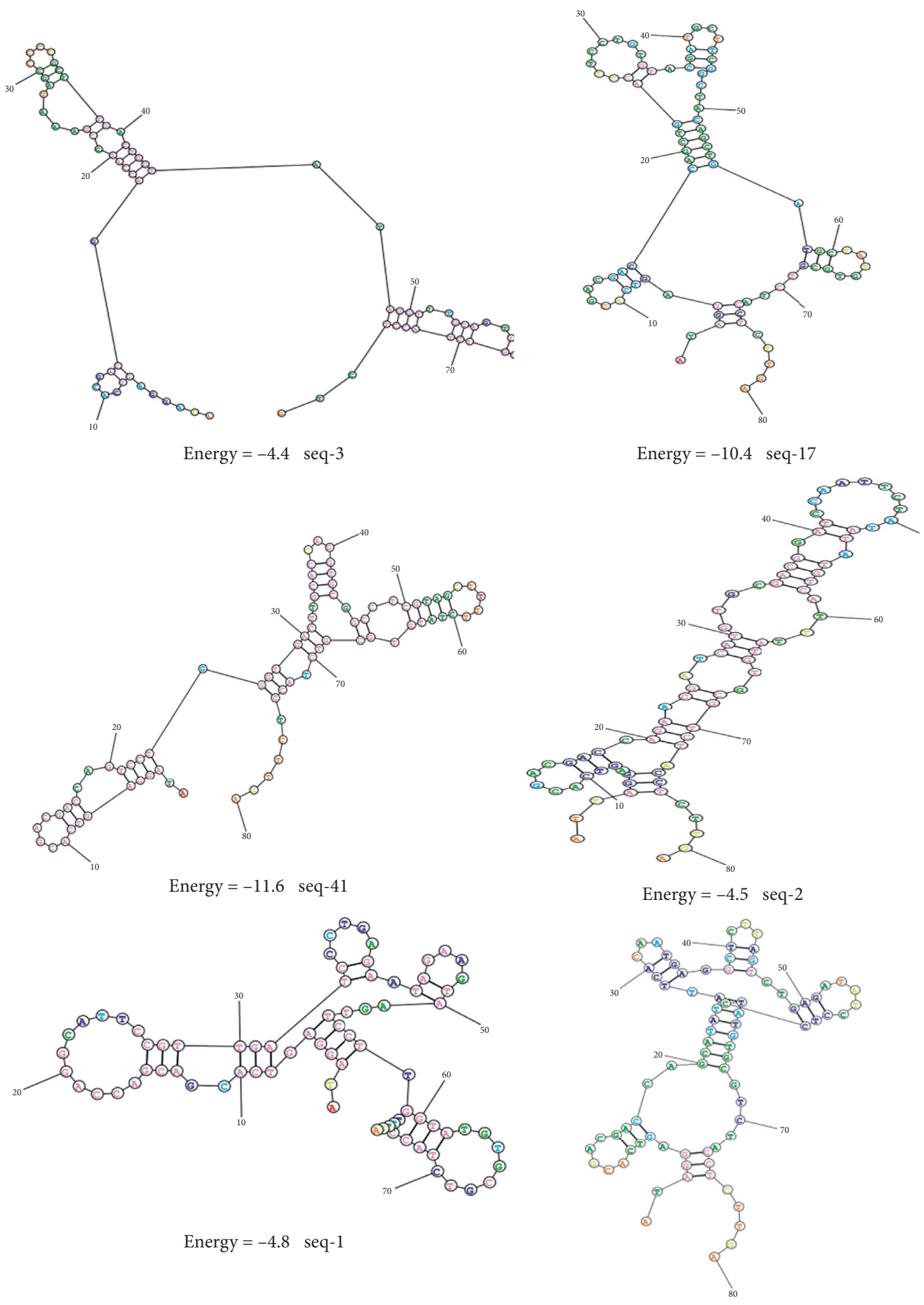

Energy $=-6.3$ seq- 4

Figure 3: The secondary structure and the minimum free energy values (dG) of the six candidate aptamers. The secondary structure and dG were predicted and generated using online RNA Structure software. The colors represent the probability $(p)$ that the base pair forms in the predicted structures. The higher the possibility is, the more likely the base pair forms and the more correct the secondary structure is (red: $p \geq 99 \%$, orange: $99 \%>p \geq 95 \%$; yellow: $95 \%>p \geq 90 \%$; green: $90 \%>p \geq 80 \%$; light green: $80 \%>p \geq 70 \%$; light blue: $70 \%>p \geq 60 \%$; blue: $60 \%>p \geq 50 \%$; pink: $50 \%>$ p).

in Figure 7, when the aptamer concentration was in the range of $0-1.0 \mu \mathrm{M}$, the absorbance value increases obviously and the color changes from gray to purple-gray, which indicated that aptamer was not enough to protect the aggregation and precipitation of gold nanoparticles under high salt concentration. When the aptamer 


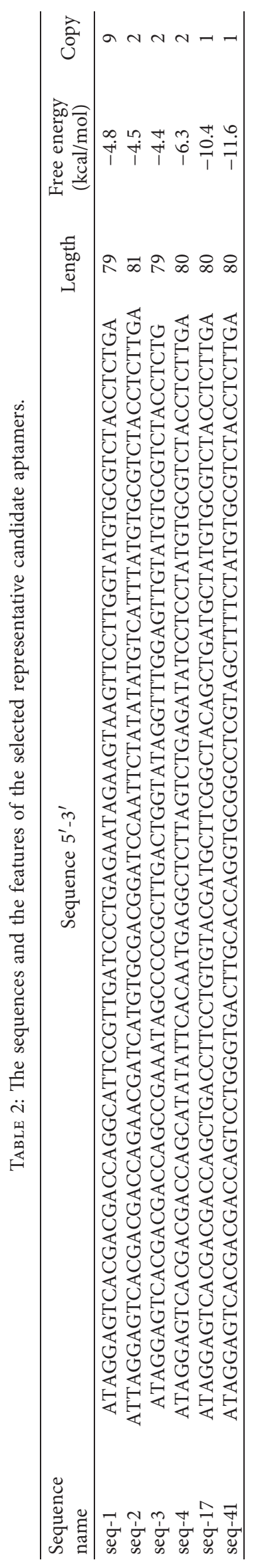


TABLE 3: Equilibrium dissociation constant $\left(K_{d}\right)$ of the six selected candidate aptamers.

\begin{tabular}{llr}
\hline Sequence & $K_{d}(\mathrm{nM})$ & $R^{2}$ \\
seq-1 & $37.94 \pm 0.1445$ & 0.9841 \\
seq-2 & $130.1 \pm 0.2984$ & 0.9712 \\
seq-3 & $135.1 \pm 0.4183$ & 0.9796 \\
seq-4 & $57.16 \pm 0.2055$ & 0.9622 \\
seq-17 & $98.03 \pm 0.2510$ & 0.9783 \\
seq-41 & $31.17 \pm 0.1070$ & 0.9857 \\
\hline
\end{tabular}
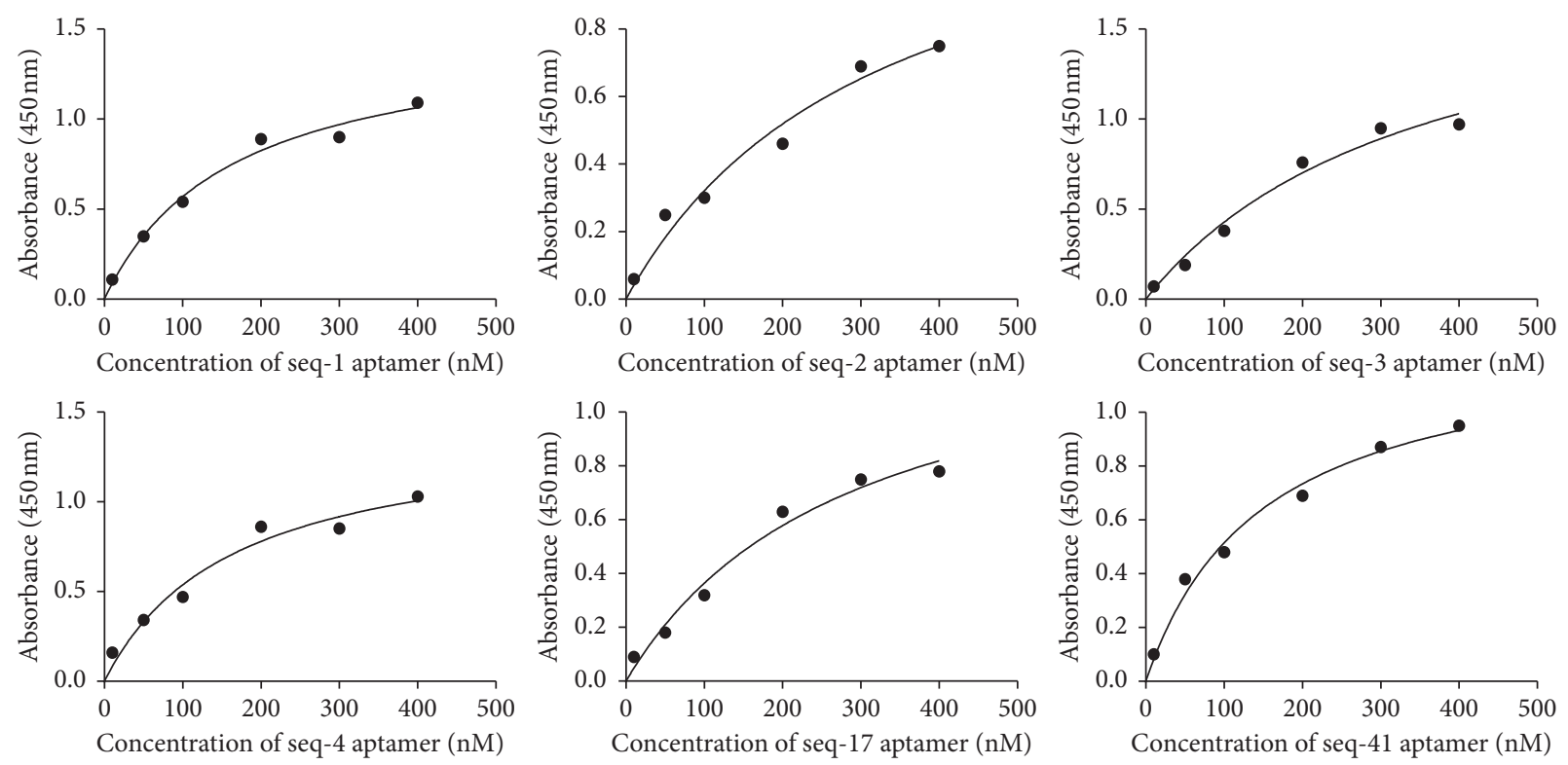

FIGURE 4: Binding affinity of the six selected candidate aptamers to ABL. Nonlinear regression analysis was performed using GraphPad Prism 6.01.

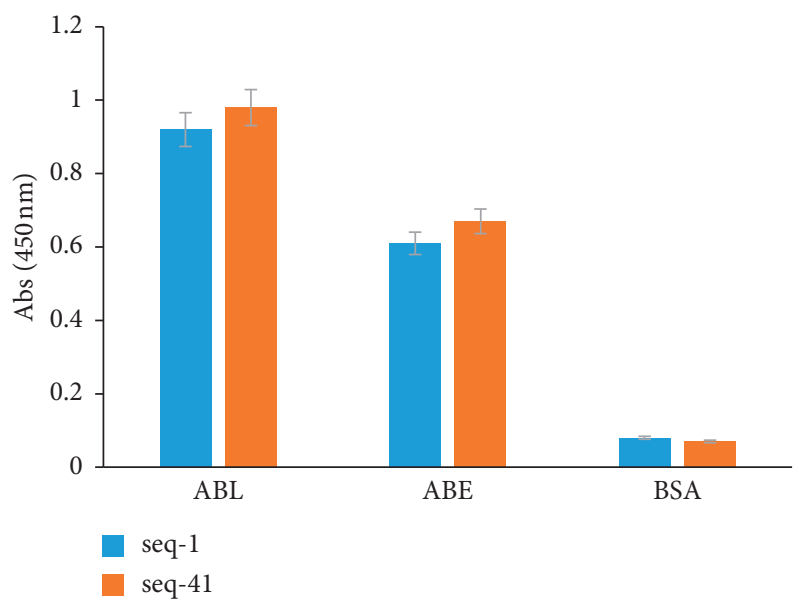

FIGURE 5: Specificity of the selected candidate aptamers seq-1 and seq-41 towards ABL. The absorbance values of seq- 41 and seq- 1 were measured at $450 \mathrm{~nm}$. ABL, ABE, and BSA are the abbreviations of Agaricus bisporus lectin, Agaricus bisporus extract, and bovine serum albumin, respectively.

concentration was equal to or higher than $2 \mu \mathrm{M}$, the color of colloidal gold solution changed from purple-gray to red, and the absorption value tended to be stable. These

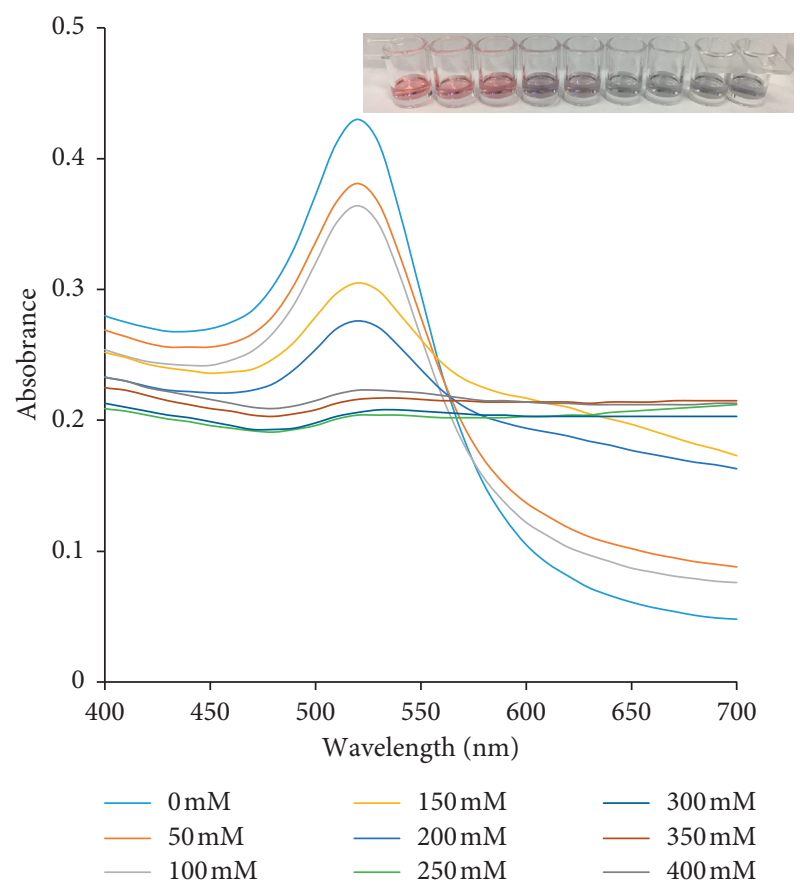

Figure 6: Optimization of the $\mathrm{NaCl}$ concentration. The embedded image showed the color changes (from left to right) with the increase of the $\mathrm{NaCl}$ concentration. 


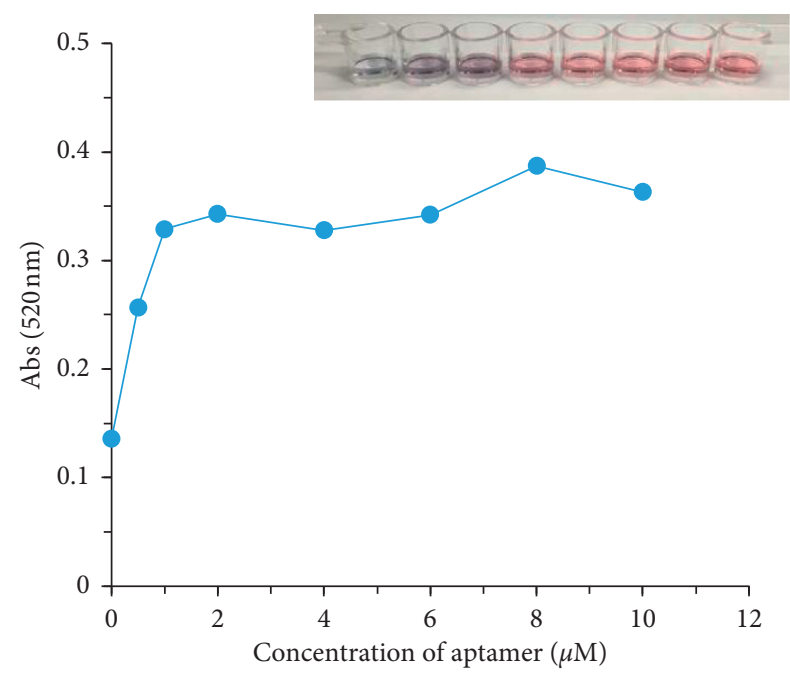

Figure 7: Optimization of aptamer concentration. The embedded image showed the color changes (from left to right) with the increase of the aptamer concentration.

evidences suggested that $2 \mu \mathrm{M}$ aptamer was appropriate for further experiments.

3.4.3. Setting Up a Standard Curve of the Aptamer-AuNPs Colorimetric Method for Detection of $A B L$. In the aptamerAuNPs colorimetric system, ABL and AuNPs competitively bind to aptamers. Under the condition of a high concentration salt solution, lectin binds with aptamer, thus releasing the free AuNPs and exposing them to the high salt solution to aggregate and precipitate. Meanwhile, the color change of AuNPs can be observed by naked eyes and monitored by UV-vis spectroscopy. As shown in Figure 8, with the increase of $\mathrm{ABL}$ concentration, the ratio of absorbance A620/A520 gradually increased, revealing that there was a good linear relationship between them. Based on these results, a standard curve was generated (Figure 8). The linear regression equation was $y=0.4146 x+0.4307$ $\left(R^{2}=0.9964\right)$ within a linear range of $0.08-1.70 \mu \mathrm{g} / \mathrm{mL}$ and the limit of detection of this method can reach $0.062 \mu \mathrm{g} / \mathrm{mL}$. The limit of detection (LOD) was calculated according to the definition of LOD $\left(\mathrm{LOD}=3 \mathrm{~S}_{\mathrm{b}} / \mathrm{m}, n=9 ; \mathrm{S}_{\mathrm{b}}\right.$ : the blank standard deviation; $\mathrm{m}$ : the slope of the linear regression equation) in a standard solution. The results demonstrated that the colorimetric method could be used to quantitatively detect ABL. Currently the main method for determination of $\mathrm{ABL}$ activity (or $\mathrm{ABL}$ concentration) is the hemagglutination activity test. According to the ABL information provided by Sigma-Aldrich, the activity of ABL is defined as the lowest concentration to agglutinate a $2 \%$ suspension of human blood group $\mathrm{O}$ erythrocytes after $1 \mathrm{~h}$ incubation at $25^{\circ} \mathrm{C}$. Agglutination activity is usually expressed in $\mu \mathrm{g} / \mathrm{mL}$ of lectin and is determined from serial dilutions in phosphate buffered saline, $\mathrm{pH} 6.8$, of a $1 \mathrm{mg} / \mathrm{mL}$ solution (https://www. sigmaaldrich.com/catalog/product/sigma/15640?lang=zh \&region $=\mathrm{CN}$ ). That is to say, the limit of detection for $\mathrm{ABL}$ is usually at the level of $\mu \mathrm{g} / \mathrm{mL}$. EY Laboratories, Inc., USA, states on its web page (http://eylabs.com/agaricus-bisporus/

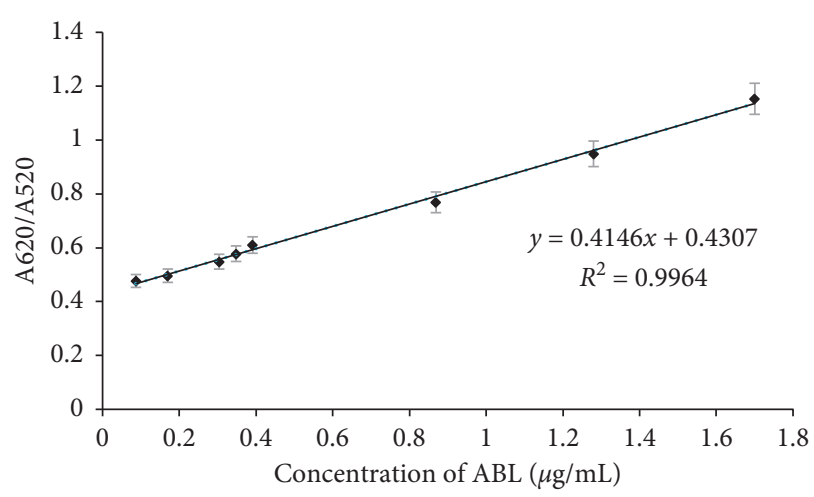

Figure 8: A standard curve of the aptamer-AuNPs colorimetric method for detection ABL. The ratio of A620/A520 has a linear relationship with the $\mathrm{ABL}$ concentrations.

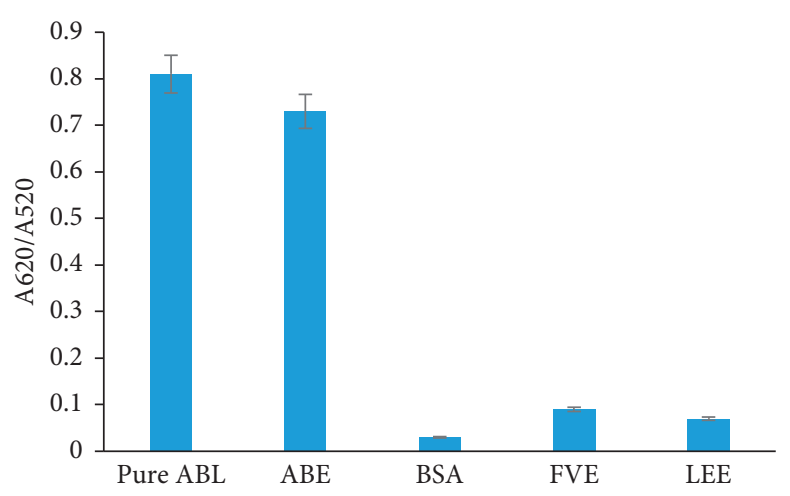

FIgURE 9: The specificity of the aptamer-AuNPs colorimetric detection method. Five different samples were tested by the aptamerAuNPs colorimetric method. ABL, ABE, BSA, FVE, and LEE are the abbreviations of Agaricus bisporus lectin, Agaricus bisporus extract, bovine serum albumin, Flammulina velutipes extract, and Lentinus edodes extract, respectively.

\#.Xy4fQv6JiUl) that $5-10 \mu \mathrm{g} / \mathrm{mL}$ ABL causes agglutination of type A or B erythrocytes and $2 \mu \mathrm{g} / \mathrm{mL}$ will agglutinate type $\mathrm{O}$ erythrocytes. Alborés et al. reported that the minimum concentration of Agaricus bisporus lectin detected by mouse erythrocytes with hemagglutination test was $7.8 \mu \mathrm{g} / \mathrm{mL}$ [33]. Comparing to these facts, the detection limit of aptamerAuNPs colorimetric method constructed in this study is about one hundred times as high as that of the hemagglutination method, which indicated that the method had much high sensitivity. Moreover, no one has reported the isolation of $\mathrm{ABL}$ aptamer as well as the ABL assay method based on the aptamer of ABL. Consequently, as we know, this is the first report about the establishment of the aptamer-AuNPs colorimetric method for detection of ABL.

\subsubsection{Specificity Verification of the Aptamer-AuNPs Color-} imetric Method for Detection of $A B L$. Some foods consumed daily may contain more than one kind of mushrooms. To explore the selectivity of the aptamer-AuNPs colorimetric method for detection of $\mathrm{ABL}$, the standard $\mathrm{ABL}$, lectin extracts from Agaricus bisporus, Flammulina velutipes, and Lentinus edodes were tested with the method. The results 
showed that the ratio of A620/A520 of standard ABL and fresh $A B L$ extract $(\mathrm{ABE})$ was dramatically higher than that of other samples, highly indicating that this method can be applied to the detection of ABL with high specificity and sensitivity (Figure 9). This may provide a potential assay method for detection of ABL from foods and agricultural products.

\section{Conclusions}

In conclusion, in this study, aptamer seq-41 was selected from the ssDNA pool after 13 SELEX selection rounds and it was proved that seq- 41 had high affinity and specificity to ABL. Based on seq-41, an aptamer-AuNPs colorimetric method for the qualitative and quantitative detection of $\mathrm{ABL}$ was established. Compared to the hemagglutination assay method, this method has high sensitivity and specificity, which provides a reference for the future research and development of the ABL detection method.

\section{Data Availability}

All data generated or analyzed during this study are included in this article.

\section{Conflicts of Interest}

The authors declare that they have no conflicts of interest.

\section{Authors' Contributions}

Hao Gao and Yiling Tian contributed equally to this manuscript.

\section{Acknowledgments}

The authors greatly appreciate Professor Wenyi Kang for providing them the ABL samples. This study was financially supported by National Key Research and Development Program of China (Project no. 2018YFD0400200).

\section{References}

[1] W. T. Iamaya, R. R. Tjandrawinta, and H. Rachmawati, "Lectins from the edible mushroom Agaricus bisporus and their therapeutic potentials," Molecules, vol. 25, no. 10, p. 2368, 2020.

[2] L. G. Yu, D. G. Fernig, M. R. H. White et al., "Edible mushroom (Agaricus bisporus) lectin, which reversibly inhibits epithelial cell proliferation, blocks nuclear localization sequence-dependent nuclear protein import," Journal of Biological Chemistry, vol. 274, no. 8, pp. 4890-4899, 1999.

[3] X. Xu, H. Yan, J. Chen, and X. Zhang, "Bioactive proteins from mushrooms," Biotechnology Advances, vol. 29, no. 6, pp. 667-674, 2011.

[4] M. Hassan, R. Rouf, E. Tiralongo, T. May, and J. Tiralongo, "Mushroom lectins: specificity, structure and bioactivity relevant to human disease," International Journal of Molecular Science, vol. 16, no. 4, pp. 7802-7838, 2015.

[5] M. E. Carrizo, S. Capaldi, M. Perduca, F. J. Irazoqui, G. A. Nores, and H. L. Monaco, "The antineoplastic lectin of the common edible mushroom (Agaricus bisporus) has two binding sites, each specific for a different configuration at a single epimeric hydroxyl," Journal of Biological Chemistry, vol. 280, no. 11, pp. 10614-10623, 2005.

[6] H. Kawagishi, "Mushroom lectins," Food Reviews International, vol. 11, no. 1, pp. 63-68, 1995.

[7] A. F. S. Santos, T. H. Silva, M. D. C. Napoleão, P. M. G. Paiva, M. T. S. Correia, and L. C. B. B. Coelho, "Lectins: function, structure, biological properties and potential applications," Current Topics in Peptide Protein Research, vol. 15, pp. 41-62, 2014.

[8] I. M. Vasconcelos and J. T. A. Oliveira, "Antinutritional properties of plant lectins," Toxicon, vol. 44, no. 4, pp. 385-403, 2004.

[9] K. Pender and L. Pruimboom, "The dietary intake of wheat and other cereal grains and their role in inflammation," Nutrients, vol. 5, pp. 771-787, 2013.

[10] J. Pohleven, N. A. Obermajer, J. Sabotič et al., "Purification, characterization and cloning of a ricin B-like lectin from mushroom Clitocybe nebularis with antiproliferative activity against human leukemic T cells," Biochemistry Biophys Acta, vol. 1790, no. 3, pp. 173-181, 2009.

[11] X. Sun, Y. Ye, S. He et al., "A novel oriented antibody immobilization based voltammetric immunosensor for allergenic activity detection of lectin in kidney bean by using AuNPs-PEI-MWCNTs modified electrode," Biosensors and Bioelectronics, vol. 143, Article ID 111607, 2019.

[12] I. Güll, M. Wirth, and F. Gabor, "Development of a sensitive and reliable ELISA for quantification of wheat germ agglutinin," Journal of Immunological Methods, vol. 318, no. 1-2, pp. 20-29, 2007.

[13] C. Rizzi, L. Galeoto, G. Zoccatelli, S. Vincenzi, R. Chignola, and A. D. B. Peruffo, "Active soybean lectin in foods: quantitative determination by ELISA using immobilised asialofetuin," Food Research International, vol. 36, no. 8, pp. 815-821, 2003.

[14] M. Darmostuk, S. Rimpelová, H. Gbelcová, and T. Ruml, "Current approaches in SELEX: an update to aptamer selection technology," Biotechnology Advances, vol. 33, no. 6, Article ID S0734975015000336, 2015.

[15] Y. Zhong, J. Y. Zhao, J. Z. Li, X. Liao, and F. L. Chen, "Advances of aptamers screened by cell-SELEX in selection procedure, cancer diagnostics and therapeutics," Analytical Biochemistry, vol. 598, Article ID 113620, 2020.

[16] A. D. Ellington and J. W. Szostak, "In vitro selection of RNA molecules that bind specific ligands," Nature, vol. 346, no. 6287 , pp. $818-822,1990$.

[17] C. Tuerk and L. Gold, "Systematic evolution of ligands by exponential enrichment: RNA ligands to bacteriophage T4 DNA polymerase," Science, vol. 249, no. 4968, pp. 505-510, 1990.

[18] S. Y. Toh, M. Citartan, S. C. B. Gopinath, and T. H. Tang, "Aptamers as a replacement for antibodies in enzyme-linked immunosorbent assay," Biosensors and Bioelectronics, vol. 64, pp. 392-403, 2015.

[19] P. Bayat, R. Nosrati, M. Alibolandi et al., "SELEX methods on the road to protein targeting with nucleic acid aptamers," Biochimie, vol. 154, pp. 132-155, 2018.

[20] F. Tian, J. Zhou, R. Fu, Y. Cui, and Y. He, "Multicolor colorimetric detection of ochratoxin a via structure-switching aptamer and enzyme-induced metallization of gold nanorods," Food Chemistry, vol. 320, Article ID 126607, 2020.

[21] J. Q. Hu, R. N. Huang, Y. Wang et al., "Research progress on utilization of nucleic acid aptamer in detection for foodborne 
pathogenic bacteria," Science and Technology of Food Industry, vol. 40, no. 9, pp. 315-322, 2019.

[22] Q. Fang, Y. Li, X. Miao et al., "Sensitive detection of antibiotics using aptamer conformation cooperated enzyme-assisted SERS technology," The Analyst, vol. 144, no. 11, pp. 3649-3658, 2019.

[23] L. Farzin, M. Shamsipur, and S. Sheibani, "A review: aptamerbased analytical strategies using the nanomaterials for environmental and human monitoring of toxic heavy metals," Talanta, vol. 174, no. 11, pp. 619-627, 2017.

[24] C. C. Chang, C. P. Chen, T. H. Wu, C. H. Yang, C. W. Lin, and C.Y. Chen, "Gold nanoparticle-based colorimetric strategies for chemical and biological sensing applications," Nanomaterials, vol. 9, no. 6, p. 861, 2019.

[25] S. Sharifi, S. Z. Vahed, E. Ahmadian et al., "Detection of pathogenic bacteria via nanomaterials-modified aptasensors," Biosensors and Bioelectronics, vol. 150, Article ID 111933, 2020.

[26] J. Du, J. Hong, C. Xu et al., "Screening and identification of ssDNA aptamer for human GP73," Biomed Research International, vol. 2015, Article ID 610281, 2015.

[27] G. Frens, "Controlled nucleation for the regulation of the particle size in monodisperse gold suspensions," Nature Physical Science, vol. 241, no. 105, pp. 20-22, 1973.

[28] C. Wang, M. Zhang, G. Yang et al., "Single-stranded DNA aptamers that bind differentiated but not parental cells: subtractive systematic evolution of ligands by exponential enrichment," Journal of Biotechnology, vol. 102, no. 1, pp. 15-22, 2003.

[29] H. Chen, C. H. Yuan, Y. F. Yang et al., "Subtractive cellSELEX selection of DNA aptamers binding specifically and selectively to Hepatocellular carcinoma cells with high metastatic potential," BioMed Research International, vol. 2016, Article ID 5735869, 2016.

[30] L. P. Zhao, G. Yang, X. M. Zhang, and F. Qu, "Development of aptamer screening against proteins and its applications," Chinese Journal of Analytical Chemistry, vol. 48, no. 5, pp. 560-572, 2020.

[31] T. Yang, Z. Luo, Y. Tian, C. Qian, and Y. Duan, "Design strategies of AuNPs-based nucleic acid colorimetric biosensors," TrAC Trends in Analytical Chemistry, vol. 124, Article ID 115795, 2020.

[32] H. Wei, B. Li, J. Li, E. Wang, and S. Dong, "Simple and sensitive aptamer-based colorimetric sensing of protein using unmodified gold nanoparticle probes," Chemical Communications, vol. 36, no. 36, pp. 3735-3737, 2007.

[33] S. Albors, P. Mora, M. P. Cerdeiras, and L. F. Fraguas, "Screening for lectins from basidiomycetes and isolation of Punctularia atropurpurascens lectin," Journal of Basic Microbiology, vol. 54, no. 2, pp. 89-96, 2014. 